\title{
Microbuckle tunnelling in fibre composites
}

\author{
N.A. Fleck*, L.G. Zhao \\ Department of Engineering, Cambridge University, Trumpington St., Cambridge CB2 1PZ, UK
}

Received 3 June 1999; received in revised form 9 November 1999

\begin{abstract}
The propagation of compressive failure in multi-directional composite laminates is modelled by the tunnelling of a microbuckle within the load-bearing axial plies, with concomitant delamination of the neighbouring off-axis plies. The microbuckle tunnels at its tip in a crack-like mode III manner, and the steady state tunnelling stress is estimated by calculating the energy difference between the upstream unbuckled state and the downstream buckled state. The downstream state is analysed in detail using a plane strain analysis of a microbuckle with delaminations from its tips. In the downstream 2D problem, microbuckling of the axial plies is represented by the generation of an inclined mode II crack, with an associated microbuckling tip toughness and a constant sliding stress across its flanks. The delaminations at the interface between the axial and adjacent off-axis plies are idealised as traction-free mixed-mode interfacial cracks. Predictions of the steady-state tunnelling stress are obtained for an isotropic solid by solving an integral equation and by the finite element method; finite element techniques are then used to solve the tunnelling problem for an orthotropic solid and for a cross-ply laminate. For each case, the tunnelling stress and the delamination crack length are obtained as functions of the ratio of delamination to microbuckle toughness, and of the inclination of the microbuckle band. The tunnelling stress provides a useful lower bound for the compressive strength of a thick laminated structure. (C) 2000 Elsevier Science Ltd. All rights reserved.
\end{abstract}

Keywords: A. Buckling; A. Energy release rate; B. Fibre-reinforced composite material; C. Finite elements

\footnotetext{
* Corresponding author. Tel.: + 44-01223-332650; fax: +44-01223-332662.

E-mail address: nafl@eng.cam.ac.uk (N.A. Fleck).
} 


\section{Introduction}

Long fibre composites, such as carbon fibres in an epoxy matrix, are widely used in laminated sheet form in the transport industries to build light, strong structures. They possess excellent tensile strengths resulting from the high strength of the fibres. However, they fail in compression at a stress level of about $60 \%$ of their tensile strength. Thus, a composite beam in bending may fail in compression rather than in tension.

A common compressive failure mode for long fibre-polymer matrix composites is imperfection-sensitive plastic microbuckling: misaligned fibres of the loadingbearing $0^{\circ}$ plies of the laminate rotate within a narrow band under the applied axial load, and lead to an instability. As the fibres rotate, the resolved shear stress along the local fibre direction increases (a geometric softening effect) in competition with the strain hardening of the matrix. The instability is analogous to the necking of a tensile bar wherein the rate of geometric softening eventually outweighs the strain hardening rate of the material. We shall refer to the compressive strength of the laminate due to this shear instability from a patch of pre-existing fibre waviness as the initiation strength of the laminate.

Plastic microbuckling has been investigated extensively in recent years (for example, Moran et al., 1995; Kyriakides et al., 1995; Kyriakides and Ruff, 1997; Hsu et al., 1998; Vogler and Kyriakides, 1997; Schapery, 1995; Jensen and Christoffersen, 1997; also, see the reviews of Schultheisz and Waas (1996), Waas and Schultheisz (1996), and Fleck (1997)). Analytical solutions have been obtained by Budiansky and Fleck (1993) for the one-dimensional case of an infinite band of initial fibre waviness. The composite is ascribed an initial imperfection in the form of a one dimensional infinite band of initial fibre misalignment, as sketched in Fig. 1a. Such a model gives useful insight into the relation between the initiation strength and the initial fibre misalignment angle (Fleck et al., 1995), and can also be used to reveal the post-collapse phenomenon of steady-state axial broadening of a kink band under constant applied stress (Budiansky et al., 1998; Jensen, 1999), as observed by Moran et al. (1995) in experiments on IM7/PEEK composites and by Vogler and Kyriakides (1997) on AS4/PEEK composites. To a good approximation, fibre rotation arrests within the broadening band when the volumetric strain equals zero. Thus, the broadening band results in the relative sliding of the composite above and below the kink band, giving it the character of a shear cohesive zone with a constant shear traction $\tau_{\mathrm{b}}$. A more detailed description of the collapse sequence is given below, for the case of a unidirectional composite containing an infinite band of initially misaligned fibres.

Consider the collapse response for the geometry given in Fig. 1a, under an axial stress $\sigma^{\infty}$. Assume that the fibres are inextensional, and are uniformly misaligned by a constant angle $\bar{\phi}$ within a band of width $w$ in the stress-free initial configuration. The normal to the band is taken to be at an angle $\beta$ to the fibre direction. The deformation mode is one of fibre rotation within the band, giving rise to an end shortening $\Delta$. The fibres are assumed to possess a finite bending resistance so that the additional fibre rotation $\phi$ under load is continuous 
spatially. This fibre rotation induces in-plane shear parallel to the fibre direction and direct straining transverse to the fibres, when the composite is subjected to a remote axial stress $\sigma^{\infty}$. Fibres within the band attain large rotations (of up to about $60^{\circ}$ ) and so a non-linear constitutive response is required for the smearedout behaviour of the composite within the band. Initially, fibre rotation and the associated overall shortening by $\Delta$ occurs under an increasing stress $\sigma^{\infty}$ as indicated by a typical point A of Fig. 1b. With continued fibre rotation the applied stress $\sigma^{\infty}$ goes through a maximum (point B) after a few degrees of fibre rotation. Beyond maximum load, fibre rotation continues and the band continues to broaden, see point $\mathrm{C}$ of Fig. 1b. Eventually, the matrix strain hardens sufficiently within the band for the material to 'lock-up' and continued end shortening of the structure is due to broadening of the band in the axial direction at a constant value of remote stress $\sigma_{\mathrm{b}}$, point $\mathrm{D}$. It is found experimentally that the locked-up state within the band is associated with a state of zero volumetric strain within the band: as the fibres rotate they first lead to dilation within the band, followed by compaction until lock-up occurs at $\phi \approx 2 \beta$ (Chaplin (1977), Evans and Adler (1978) and Sivashanker et al. (1996)). The response for a perfect composite, $\bar{\phi}=0$, is included in Fig. 1b: an elastic shear bifurcation occurs at a peak axial stress equal to the in-plane shear modulus $G$, with infinite initial wavelength, as first derived by Rosen (1965). The subsequent collapse response merges with that for the imperfect composite after a few degrees of fibre rotation.

(a)

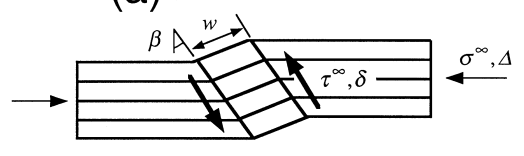

(b)

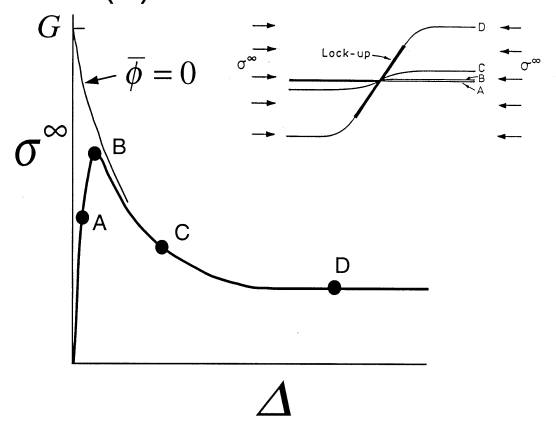

(c)

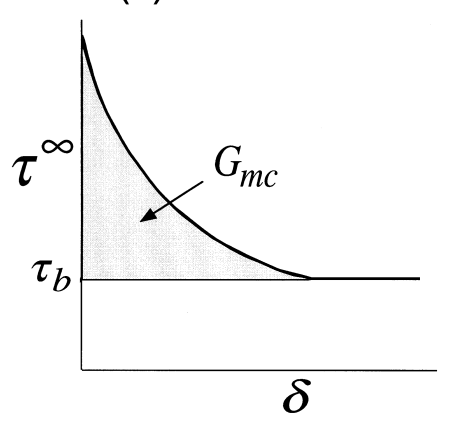

Fig. 1. (a) Microbuckling from an infinite band of fibre misalignment, of magnitude $\bar{\phi}$. (b) Axial collapse response of infinite band, for the cases of a finite initial imperfection and for a perfect composite. (c) Collapse response of a perfect composite, expressed in terms of the shear traction on the inclined microbuckle band, and the work-conjugate sliding displacement $\delta$. 
The above collapse response has been calculated in detail by Budiansky et al. (1998) using large rotation, 1D bending theory. Upon making the assumption of fibre inextensionability, the kinematics were parameterised by the fibre rotation $\phi$ along the fibre length. Band broadening results in the progressive relative sliding of material on each side of the microbuckle band, in a direction parallel to the band inclination $\beta$, without dilatation. Thus, the applied loading $\sigma^{\infty}$ versus $\Delta$ collapse response can be rephrased in terms of the resolved shear traction $\tau^{\infty}=$ $\sigma^{\infty} \cos \beta \sin \beta$ parallel to the band, and the work-conjugate relative sliding displacement $\delta$ across the band, as sketched in Fig. 1a. Thus, the microbuckle can be treated as a cohesive zone, with a shear traction $\tau^{\infty}$ versus crack sliding displacement $\delta$ response, as sketched in Fig. 1c for the case of a perfect composite with $\bar{\phi}=0$. We shall use this $\tau^{\infty}-\delta$ cohesive zone law in the calculation of the steady state tunnelling stress of a microbuckle, following the arguments outlined by Fleck (1997). The subsequent analysis is simplified considerably by partitioning the area under the $\tau^{\infty}-\delta$ curve into two parts: (i) the area under a constant band broadening stress $\tau_{\mathrm{b}}=\sigma_{\mathrm{b}} \cos \beta \sin \beta$, and (ii) a finite remainder $G_{\mathrm{mc}}$, as sketched in Fig. 1c. This partitioning allows us to treat the propagating microbuckle as a crack carrying a constant crack bridging shear traction $\tau_{\mathrm{b}}$ along its flanks, with a tip toughness $G_{\mathrm{mc}}$. A similar strategy has been adopted by Palmer and Rice (1973) in the study of mode II shear faults in soils and rocks.

\subsection{Analysis of tunnelling using a two dimensional crack model}

We shall now use the above ideas to model the observed 3D tunnelling of a microbuckle in the axial plies of a multi-directional laminate. Experiments by Soutis et al. (1991); Soutis et al. (1993) and Fleck et al. (1997) show that the compressive failure of multi-directional laminates is typically by out-of-plane microbuckling of the load-bearing $0^{\circ}$ plies, accompanied by delaminations between the $0^{\circ}$ and adjacent off-axis plies. The microbuckle together with the delaminations can propagate through the $0^{\circ}$ ply of a thick composites by a $3 \mathrm{D}$ tunnelling mechanism, as sketched in Fig. 2a. In the vicinity of its tip the microbuckle resembles a sliding mode III crack, but downstream from its tip the deformation state can be approximated by a plane strain mode II crack, with delaminations emanating from each tip.

At large tunnel lengths, the microbuckle maintains a fixed frontal shape and with a fixed mode III sliding displacement downstream of its tip. In this steady state, tunnelling occurs at a constant values of the axial stress $\sigma_{\mathrm{ss}}$. On treating the microbuckle as a bridged crack, the energy release rate per unit advance of the tunnelling crack can be calculated from plane-strain elasticity solutions by considering the stress states both upstream and downstream of the crack front (Hutchinson and Suo, 1992; Beuth, 1992; Chan et al., 1993; Ho and Suo, 1993). Downstream, the tunnelling microbuckle is represented by a $2 \mathrm{D}$ mode II crack with an anti-symmetric pair of delamination cracks, as illustrated in Fig. 2b. Here, $\sigma^{\infty}$ is the remote compressive stress, $\beta$ and $2 a$ are the inclination and length of the mode II crack, and $l$ is the length of each delamination. It is assumed that the 
sliding of the mode II crack is resisted by a constant bridging shear traction $\tau_{\mathrm{b}}$ imposed by the kinked fibres, and that the creation of the microbuckle results in an energy dissipation per unit area equal to the toughness $G_{\mathrm{mc}}$, as sketched in Fig. 1c. The origins of this toughness are the energy stored within the bent elastic fibres and the sheared matrix between the fibres at the boundaries of a microbuckle band, as calculated by Fleck (1997) and Budiansky et al. (1998).

The calculation of the tunnelling stress is simplified considerably by neglecting the dependence of the delamination toughness upon the mode mix, so that the steady state can be found by plane strain elasticity. A posteri checks of the calculated mode mix in the $2 \mathrm{D}$ problem suggest that the mode mix is remarkably constant over a wide range of delamination lengths, in support of the assumption of constant delamination toughness. However, the analysis ignores the possible existence of a mode III stress intensity factor of the delamination front at the tip of the tunnelling microbuckle: a 3D calculation would be required to determine the mode mix at the front of the advancing delamination, but this is beyond the scope of the present study.

The axial stress for the steady state tunnelling of a microbuckle has been calculated with the finite element code ABAQUS (1997) for various microbuckle inclinations $\beta$ and normalised delamination lengths, $l / a$. Considerations are given
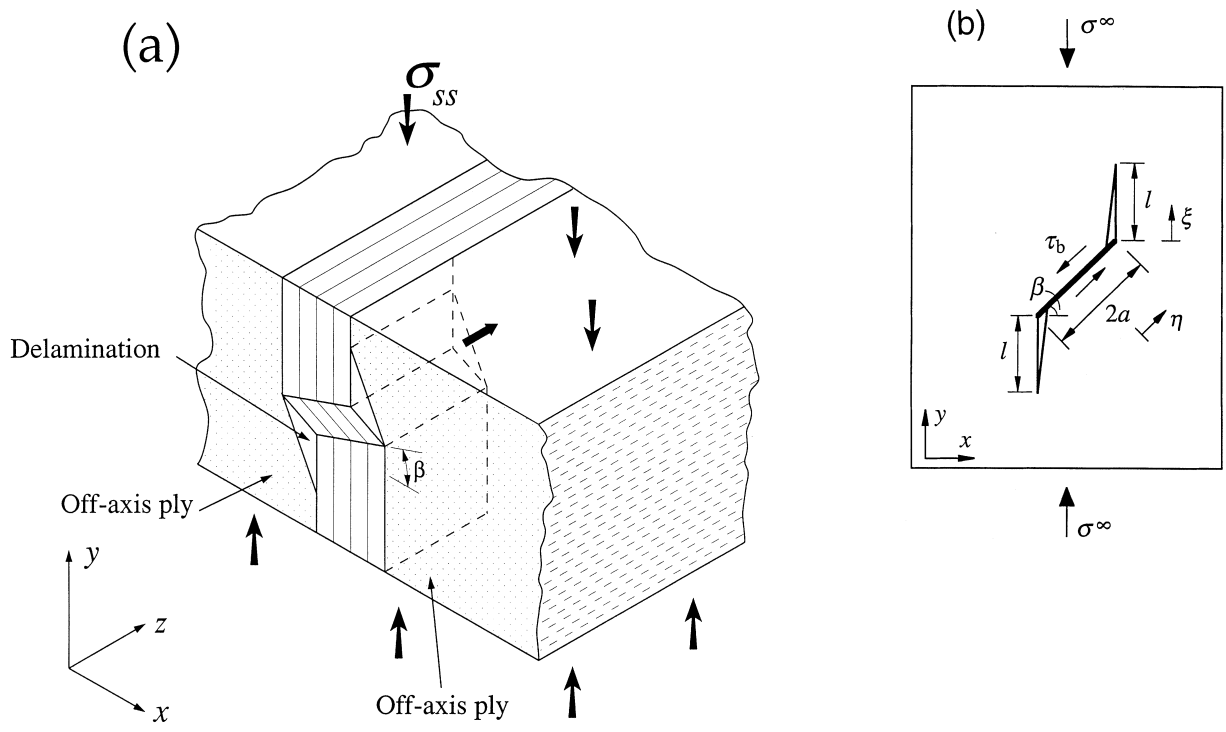

Fig. 2. (a) Sketch of a tunnelling microbuckle in the axial plies of a multi-directional laminate, with delaminations between the axial and off-axis plies. The remote compressive stress $\sigma_{\mathrm{ss}}$ is independent of the length of the tunnelling microbuckle. (b) Simulation of a microbuckle and delaminations by a plane strain mode II crack with an anti-symmetric pair of delamination cracks. 
to an isotropic solid, to orthotropic solids (to represent unidirectional laminates), and to an orthotropic bimaterial (to represent a cross-ply laminate). Independent checks on the accuracy of the finite element method have been performed: the cracks are represented by distributions of dislocations in the isotropic case, and solutions are obtained by standard integral equation techniques. For all types of laminate considered, it is found that the tunnelling stress attains a minimum for a particular length of delamination $l_{\mathrm{c}}$; at the minimum value of tunnelling stress the solution has the property that the energy release rate for continued delamination downstream of the tunnelling microbuckle equals the delamination toughness. The minimum tunnelling stress and the associated delamination length $l$ are obtained as functions of the ratio of delamination toughness to microbuckle toughness for the various classes of composite (isotropic, orthotropic and cross-ply).

\section{Tunnelling in an isotropic solid}

\subsection{Analysis}

If the fibres and matrix have approximately equal elastic constants, the problem is simplified to the case of a homogeneous isotropic solid. A related wing-crack problem has been studied by Nemat-Nasser and Horii (1982), Steif (1984) and Lauterbach and Gross (1998): they each considered crack growth from the tips of a parent mode II crack under plane deformation. For the problem shown in Fig. 2, two modes of crack extension are possible: (i) the plane strain problem of growth of mixed-mode delaminations along the interface, and (ii) the combined tunnelling of the mode II crack and the delamination cracks. Both plane strain crack growth and tunnelling are driven by the resolved shear stress component, $\tau^{\infty}=$ $\sigma^{\infty} \cos \beta \sin \beta$ along the mode II crack line, where $\sigma^{\infty}$ is the remote axial stress.

Consider an uncracked solid of unit depth, under a compressive stress $\sigma^{\infty}$. Upon introduction of a mode II sliding crack with delaminations as shown in Fig. $2 \mathrm{~b}$, the energy released $\Delta W$ per unit crack depth is

$$
\Delta W=\frac{1}{2}\left(\tau^{\infty}-\tau_{\mathrm{b}}\right) \bar{\delta} 2 a
$$

where the average crack sliding displacement $\bar{\delta}$ of the mode II crack is defined by

$$
\bar{\delta}=\frac{1}{2 a} \int_{-a}^{a} \delta(\xi) \mathrm{d} \xi
$$

Dimensional arguments allow $\bar{\delta}$ to be written as

$$
\bar{\delta}=\frac{a\left(\tau^{\infty}-\tau_{\mathrm{b}}\right)}{\bar{E}} f\left(\frac{l}{a}\right)
$$

in terms of a specified modulus $\bar{E}$ (such as the plane strain modulus in the 
isotropic case), and of a dimensionless function $f$, to be determined by subsequent analysis. On combining Eqs. (1)-(3) the energy released per unit crack depth $\Delta W$ is

$$
\Delta W=\frac{\left(\tau^{\infty}-\tau_{\mathrm{b}}\right)^{2}}{\bar{E}} a^{2} f\left(\frac{l}{a}\right)
$$

Now the plane strain energy release rate $G_{\mathrm{ps}}$ per unit advance of delamination along the interface may be expressed as

$$
G_{\mathrm{ps}}=\frac{1}{2} \frac{\partial \Delta W}{\partial \ell}
$$

Upon making use of Eq. (4), $G_{\mathrm{ps}}$ is given by

$$
G_{\mathrm{ps}}=\frac{\left(\tau^{\infty}-\tau_{\mathrm{b}}\right)^{2}}{2 \bar{E}} a f^{\prime}\left(\frac{l}{a}\right)
$$

The remote shear stress for plane strain delamination $\tau_{\mathrm{ps}}$ is obtained by equating $G_{\mathrm{ps}}$ with the delamination toughness $G_{\mathrm{dc}}$ at the appropriate mode mix, to obtain

$$
\tau_{\mathrm{ps}}-\tau_{\mathrm{b}}=\sqrt{\frac{2 \bar{E} G_{\mathrm{dc}}}{a f^{\prime}(l / a)}}
$$

It is convenient to rewrite Eq. (7) in the dimensionless form,

$$
\frac{\tau_{\mathrm{ps}}-\tau_{\mathrm{b}}}{\sqrt{\bar{E} G_{\mathrm{dc}} / a}}=\sqrt{\frac{2}{f^{\prime}(l / a)}}
$$

Now consider the alternative crack advance mode of crack tunnelling. The steadystate energy release rate, $G_{\mathrm{ss}}$, averaged over the total crack length of $2(a+l)$ is related to the elastic energy change $\Delta W$ by (Hutchinson and Suo, 1992; Ho and Suo, 1993; Chan et al., 1993):

$$
(2 a+2 l) G_{\mathrm{ss}}=\Delta W
$$

On writing the microbuckling toughness as $G_{\mathrm{mc}}$, steady state tunnelling is achieved when the energy released $\Delta W$ equals the energy absorbed by delamination and by microbuckling, viz

$$
(2 a+2 l) G_{\mathrm{ss}}=\Delta W=2 a G_{\mathrm{mc}}+2 l G_{\mathrm{dc}}
$$

On making use of Eq. (4) the remote shear stress to activate steady-state tunnelling $\tau_{\mathrm{ss}}$ is

$$
\tau_{\mathrm{ss}}-\tau_{\mathrm{b}}=\sqrt{\frac{2 \bar{E}\left(a G_{\mathrm{mc}}+l G_{\mathrm{dc}}\right)}{a^{2} f(l / a)}}
$$


or, in dimensionless form,

$$
\frac{\tau_{\mathrm{ss}}-\tau_{\mathrm{b}}}{\sqrt{\bar{E} G_{\mathrm{dc}} / a}}=\sqrt{\frac{2\left(a G_{\mathrm{mc}}+l G_{\mathrm{dc}}\right)}{a G_{\mathrm{dc}} f(l / a)}}
$$

\subsection{Numerical results and discussions}

According to the above analysis, the kernel problems are to find the energy release rate, $G_{\mathrm{ps}}$, of the delamination crack tip and the average sliding displacement, $\bar{\delta}$, of the mode II crack in order to determine $G_{\mathrm{ss}}$. For the isotropic case, calculations have been performed by both integral equation and finite element methods in order to assess the accuracy of the finite element approach.

The dislocation distribution method is described briefly in Appendix A. The underlying idea is to replace the cracks by continuous distributions of dislocations and to write the traction on the crack planes in integral equation format; the integral equations are solved numerically by adopting a Chebyshev polynomials technique (Erdogan and Gupta, 1972). From the obtained solution for the dislocation density, the Mode I and Mode II stress intensity factors, $K_{\mathrm{I}}$ and $K_{\mathrm{II}}$, and the energy release rate, $G_{\mathrm{ps}}$, at the delamination crack tip are computed by (A7). The average sliding displacement, $\bar{\delta}$, of the mode II crack is calculated from Eq. (2) together with Eq. (A8).

The ABAQUS finite element calculations made use of a mesh comprising up to 3400 elements of type 8-noded plane strain isoparametric quadrilaterals, with $3 \times$ 3 Gauss points of integration. The delamination crack geometry of Fig. $2 b$ is placed at the mid-point of the mesh, and the width and height of the mesh are chosen to exceed $40 \mathrm{l}$. The square root singularity of the delamination crack tip stress field is modelled by moving the mid-side nodes on the side adjacent to the crack tip to the quarter points for all crack tip elements. Fixed and roller boundary conditions are imposed at the two lower corner points of the mesh in order to prevent rigid body motion. The energy release rate, $G_{\mathrm{ps}}$, of the delamination crack tip is evaluated by using Parks (1974) virtual crack extension technique. The auxiliary field is taken as the singular crack tip field (Matos et al., $1989)$ to separate the stress intensity factors $K_{\mathrm{I}}$ and $K_{\mathrm{II}}$. The average sliding displacement, $\bar{\delta}$, of the mode II crack is evaluated in Eq. (2) by making use of the relative slip between the two sliding crack surfaces; the mode II crack is modelled by a pair of contact surfaces within ABAQUS and is subjected to normal and shear tractions by resolving the remote stress $\sigma^{\infty}$ onto the crack plane. The bridging stress $\tau_{\mathrm{b}}$ is taken to vanish, without loss of generality. The average tunnelling energy release rate $G_{\mathrm{ss}}$ follows directly from Eqs. (1) and (9).

Computations of $G_{\mathrm{ps}}$ and $G_{\mathrm{ss}}$ were performed for various values of $l / a$ by taking the inclination of the mode II crack to be $\beta=15^{\circ}, 30^{\circ}, 45^{\circ}$, and $60^{\circ}$. The finite element results for $G_{\mathrm{ps}}$ and $G_{\mathrm{ss}}$ are within $3 \%$ of the values obtained by the integral equation formulation. Typical results are displayed in Fig. 3a for the delamination energy release rate $G_{\mathrm{ps}}$ for a plane strain delamination crack and in 
Fig. $3 \mathrm{~b}$ for the phase angle $\psi$, where $\tan \psi \equiv K_{\mathrm{II}} / K_{\mathrm{I}}$. The parallel results for the average energy release rate for tunnelling $G_{\mathrm{ss}}$ are given in Fig. 3c. It is seen from Fig. 3a that the plane strain energy release rate $G_{\mathrm{ps}}$ drops with increasing $l / a$, implying that plane strain crack growth requires an increasing remote stress. The phase angle $\psi$ shows a small dip in value at $l / a \approx 1$, and asymptotes to a constant value at large $l / a$; as a rule of thumb the value of $\psi$ at large $l / a$ is given by $\psi \approx \beta$. The observation that $\psi$ changes little with $l / a$ justifies the assumption of taking a constant value of delamination toughness $G_{\mathrm{dc}}$ in the analysis (for example in Eq. (10)).

At an early stage of delamination, $l / a \approx 1$, the tunnelling energy release rate $G_{\mathrm{ss}}$ increases and reaches a maximum value; it then drops monotonically. The evolution of $G_{\mathrm{ps}}$ and $G_{\mathrm{ss}}$ with crack length $l$ is shown in Fig. $3 \mathrm{~d}$ for $\beta=15^{\circ}$ and $30^{\circ}$. A cross-over point is noted such that $G_{\mathrm{ps}}$ exceeds $G_{\mathrm{ss}}$ for $l / a$ less than about unity, and $G_{\mathrm{ps}}$ is less than $G_{\mathrm{ss}}$ at larger values of crack length. We note that $G_{\mathrm{ss}}$

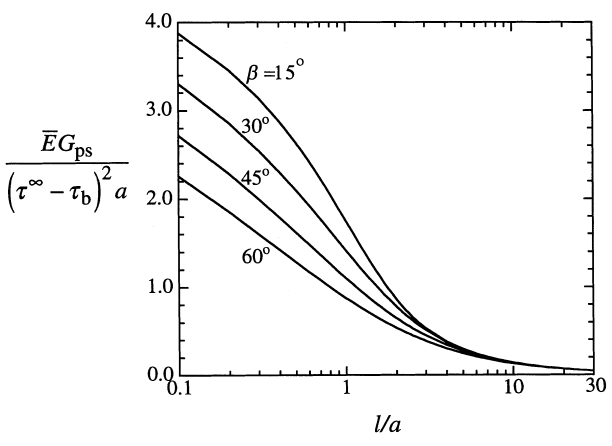

(a)

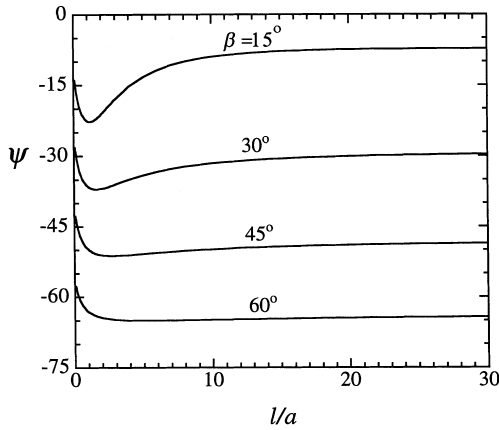

(b)

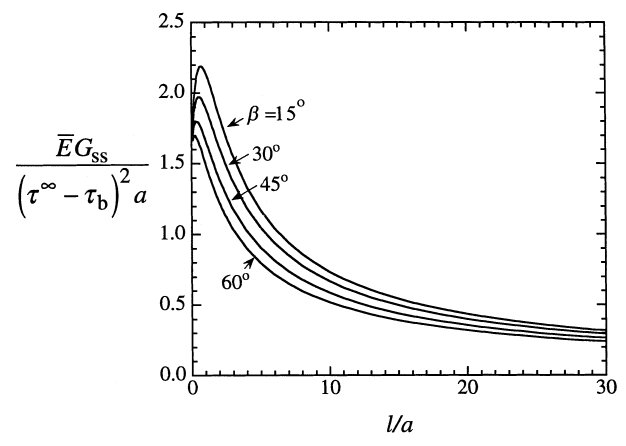

(c)

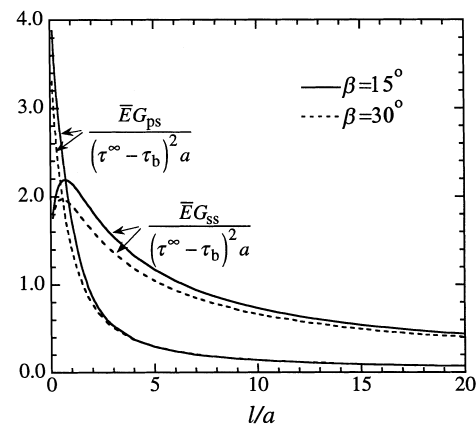

(d)

Fig. 3. Elastic solutions for a mode II crack and delaminations under remote compression. (a) Plane strain energy release rate $G_{\mathrm{ps}}$ and (b) mode mix $\psi$ for delaminations; (c) Energy release rate for steady state tunnelling $G_{\mathrm{ss}}$; (d) Comparison of $G_{\mathrm{ps}}$ and $G_{\mathrm{ss}}$. 
has a local maximum at a particular value of normalised crack length, $(l / a)_{G}$. As discussed by Beuth (1992), $G_{\mathrm{ss}}$ equals $G_{\mathrm{ps}}$ at this local maximum in $G_{\mathrm{ss}}$; this result follows directly by substitution of Eq. (9) into Eq. (5). However, it is not true that the tunnelling stress is a minimum for the choice of delamination length $l / a=$ $(l / a)_{G}$. To investigate this further, the tunnelling stress $\tau_{\mathrm{ss}}$ and the plane strain delamination stress $\tau_{\mathrm{ps}}$ are plotted in Fig. 4 against the normalised delamination length $l / a$, for selected values of $G_{\mathrm{mc}} / G_{\mathrm{dc}}$, with $\beta=30^{\circ}$. The delamination stress $\tau_{\mathrm{ps}}$ increases monotonically with $l / a$, displaying a stable crack growth response for the plane strain case. In contrast, the tunnelling stress attains a minimum value at a crack length $(l / a)_{\mathrm{c}}$. It is seen from the numerical results that $\tau_{\mathrm{ps}}$ equals $\tau_{\mathrm{ss}}$ at the location where $\tau_{\mathrm{ss}}$ is a minimum. This result can be proved as follows. The condition that $\partial \tau_{\mathrm{ss}} / \partial l=0$ in Eq. (11) gives directly the identity $\tau_{\mathrm{ss}}=\tau_{\mathrm{ps}}$, where $\tau_{\mathrm{ps}}$ has already been defined in Eq. (7).

Let us explore further the implications of the results shown in Fig. 4. For the delamination length $l / a=(l / a)_{\mathrm{c}}$ the energy release rate for continued delamination downstream of the tunnelling microbuckle equals the delamination toughness. For $l / a>(l / a)_{c}$ the remote stress required for downstream delamination $\tau_{\mathrm{ps}}$ is greater than the tunnelling stress $\tau_{\mathrm{ss}}$ and we conclude that the delamination downstream is in an unloaded state during tunnelling at $\tau^{\infty}=\tau_{\mathrm{ss}}$; this is a viable solution, but the initial choice $l / a>(l / a)_{\mathrm{c}}$ gives rise to a larger tunnelling stress $\tau_{\mathrm{ss}}$ than the minimum value at $l / a=(l / a)_{\mathrm{c}}$. For $l / a<(l / a)_{\mathrm{c}}$ the remote stress required for downstream delamination $\tau_{\mathrm{ps}}$ is less than the tunnelling stress $\tau_{\mathrm{ss}}$ and we conclude that the delamination downstream is over-loaded at $\tau^{\infty}=\tau_{\mathrm{ss}}$. Thus, stable delamination occurs at $l / a=(l / a)_{\mathrm{c}}$, and we shall assume that the associated minimum tunnelling stress is the observed value. For the special case $G_{\mathrm{mc}}=G_{\mathrm{dc}}$ the length $(l / a)_{\mathrm{c}}$ at which $\tau_{\mathrm{ss}}$ is a minimum equals the length $(l / a)_{G}$ at which $G_{\mathrm{ss}}$ is a maximum. This result may be seen by comparing the identity $\partial G_{\mathrm{ss}} / \partial l=0$ (from Eqs. (5) and (9)), with the identity $\partial \tau_{\mathrm{ss}} / \partial l=0$ (from Eq. (11)). Cox and Marshall

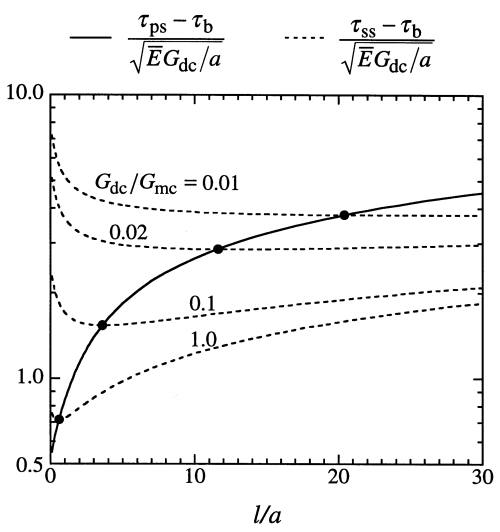

Fig. 4. Resolved stress $\tau_{\mathrm{ps}}$ for plane strain cracking compared with the resolved stress $\tau_{\mathrm{ss}}$ for steady state tunnelling, as a function of non-dimensional delamination length $l / a . \beta=30^{\circ}$. 
(1996) have outlined similar arguments for the local minimum in tunnelling stress for a mode I transverse ply crack in a laminated ceramic composite. They too show that the tunnelling crack length for which the applied stress is a minimum is different from the tunnelling length for which the tunnelling energy release rate is a maximum.

The tunnelling crack length $(l / a)_{\mathrm{c}}$ and the corresponding minimum tunnelling stress are plotted as functions of $G_{\mathrm{dc}} / G_{\mathrm{mc}}$ in Fig. $5 \mathrm{a}$ and $\mathrm{b}$ respectively, for selected values of the inclination $\beta$. The delamination length drops and the tunnelling stress increases with an increase in delamination toughness, but are relatively insensitive to the inclination $\beta$.

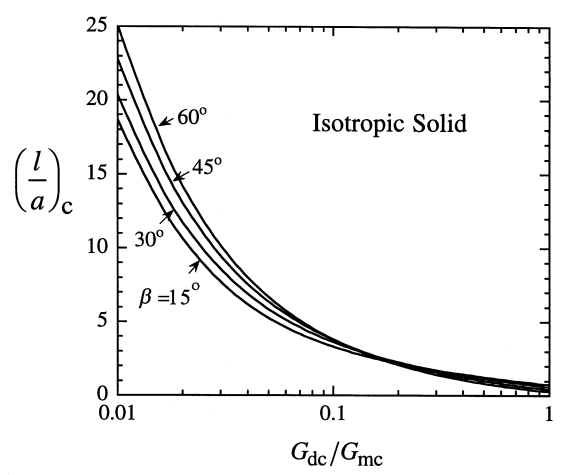

(a)

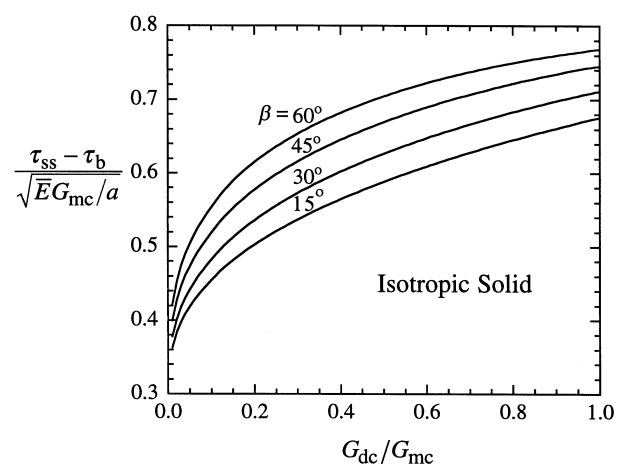

(b)

Fig. 5. (a) Delamination length and (b) minimum tunnelling stress for isotropic solid, as a function of the ratio of delamination toughness $G_{\mathrm{dc}}$ to the microbuckling toughness $G_{\mathrm{mc}}$. 


\section{Tunnelling in an orthotropic solid}

\subsection{Analysis}

For a general anisotropic material under plane strain deformation, the elastic constitutive relation has the following form (Lekhnitskii, 1963):

$$
\varepsilon_{i}=\sum_{j=1,2,6} b_{i j} \sigma_{j}, \quad i=1,2,6
$$

where

$$
\begin{aligned}
& \varepsilon_{1} \equiv \varepsilon_{x x}, \quad \varepsilon_{2} \equiv \varepsilon_{y y}, \quad \varepsilon_{6} \equiv \gamma_{x y} \\
& \sigma_{1} \equiv \sigma_{x x}, \quad \sigma_{2} \equiv \sigma_{y y}, \quad \sigma_{6} \equiv \sigma_{x y}
\end{aligned}
$$

and

$$
b_{i j}=s_{i j}-s_{i 3} s_{j 3} / s_{33}, \quad i, j=1,2,6, \quad \text { for plane strain }
$$

The axes $(x, y)$ have already been defined in Fig. 2, and $s_{i j}$ are the compliances which are related to the Young's moduli, shear moduli and Poisson's ratios of the material in the $(x, y)$ reference frame.

If the material is orthotropic and the $x$ and $y$ axes are aligned with the principal axes of the material, then there are only four independent elastic constants $b_{11}, b_{12}=b_{21}, b_{22}$, and $b_{66}$, with $b_{16}=b_{26}=0$. In this case, the non-zero compliances $s_{i j}$ are related to the engineering constants by

$$
\begin{aligned}
& s_{11}=\frac{1}{E_{x}}, \quad s_{22}=\frac{1}{E_{y}}, \quad s_{33}=\frac{1}{E_{z}} \\
& s_{12}=-\frac{v_{x y}}{E_{x}}=s_{21}=-\frac{v_{y x}}{E_{y}} \\
& s_{13}=-\frac{v_{x z}}{E_{x}}=s_{31}=-\frac{v_{z x}}{E_{z}} \\
& s_{23}=-\frac{v_{y z}}{E_{y}}=s_{32}=-\frac{v_{z y}}{E_{z}} \\
& s_{66}=\frac{1}{G_{x y}}
\end{aligned}
$$

For simply connected domains with traction boundary conditions, Suo (1990a) has shown that the stresses within an plane orthotropic solid depend on only the 
following two (rather than three) non-dimensional elastic parameters:

$$
\lambda=\frac{b_{11}}{b_{22}} \quad \text { and } \quad \rho=\frac{b_{12}+\frac{1}{2} b_{66}}{\left(b_{11} b_{22}\right)^{1 / 2}}
$$

When $\lambda=\rho=1$ the in-plane behaviour is isotropic, and when just $\lambda=1$ the solid has cubic in-plane symmetry. Positive definiteness of the strain energy density requires $\lambda>0$ and $-1<\rho<\infty$. Suo (1990a) has also shown that the number of material parameters can be further reduced to the single parameter $\rho$ by rescaling the $x$ co-ordinate to $\xi=\lambda^{1 / 4} x$. This procedure is not used in the current problem as a stretching of the $x$-axis results in a change of the crack inclination $\beta$; since only a representative sample of results are presented in the current study it is simpler and more transparent to show results for selected values of $\rho$ and $\lambda$.

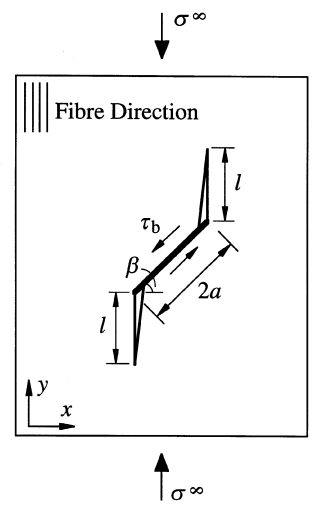

(a)

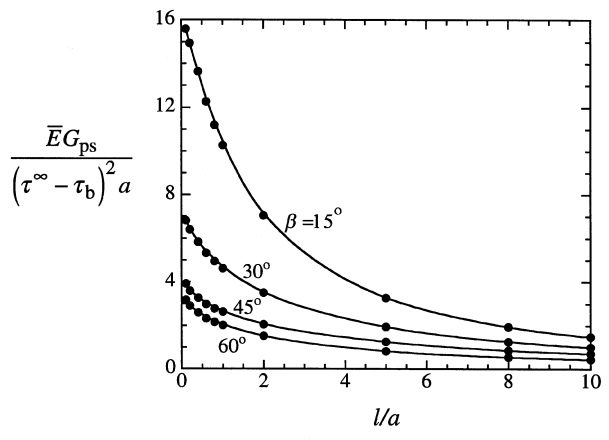

(b)

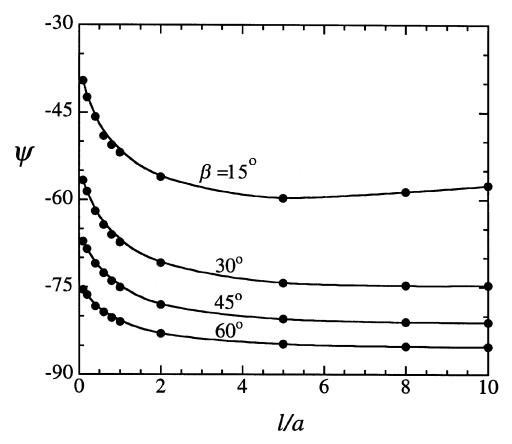

(c)

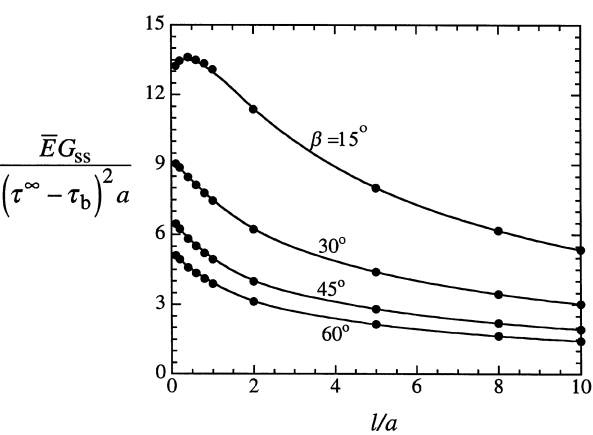

(d)

Fig. 6. Plane strain crack growth versus the tunnelling of a microbuckle in an orthotropic composite. (a) Geometry; (b) Energy release rate $G_{\mathrm{ps}}$ for the plane strain delamination crack $\left(\bar{E} \equiv 1 / b_{22}\right)$; (c) Phase angle $\psi$ for the plane strain delamination crack; (d) Tunnelling energy release rate $G_{\text {ss }}$. 
The remote stresses for plane strain delamination growth and for tunnelling propagation within a unidirectional orthotropic laminate (see Fig. 6a) are given by Eqs. (7) and (11), but now the results depend upon $\rho$ and $\lambda$ in addition to $G_{\mathrm{dc}}$, $G_{\mathrm{mc}}$ and $\beta$. For definiteness, $\bar{E}$ is given the value $1 / b_{22}$ in Eqs. (3)-(11) for the orthotropic unidirectional laminate.

\subsection{Finite element results and discussion}

Upon adopting the same meshes and calculation techniques as used in the finite element analysis for an isotropic solid, the energy release rate, $G_{\mathrm{ps}}$, at the delamination crack tip and the average sliding displacement, $\bar{\delta}$, of the mode II crack for unidirectional laminates can also be computed using the finite element code ABAQUS. The energy drop $\Delta W$ follows from Eq. (1) and the tunnelling stress from Eq. (11).

First, consider the representative case of a transversely isotropic composite material Graphite/Epoxy (T300/5208), whose properties are listed in Table 1 (Tsai, 1988). The associated values for the orthotropic parameters $\lambda$ and $\rho$ are $\lambda=13.2$ and $\rho=3.37$. The normalised energy release rate, $\bar{E} G_{\mathrm{ps}} /\left(\tau^{\infty}-\tau_{\mathrm{b}}\right)^{2} a$, at the delamination crack tip and the corresponding phase angle, $\psi=\arctan \left(K_{\mathrm{II}} / K_{\mathrm{I}}\right)$, are plotted in Fig. $6 \mathrm{~b}$ and c, respectively. The normalised tunnelling energy release rate $\bar{E} G_{\text {ss }} /\left(\tau^{\infty}-\tau_{\mathrm{b}}\right)^{2} a$ is plotted in Fig. $6 \mathrm{~d}$ for comparison. As for the isotropic case, the plane-strain energy release rate drops with increasing $l / a$, and the tunnelling energy release rate attains a local maximum at a small value of $l / a$ of less than unity. The phase angle $\psi=\arctan \left(K_{\mathrm{II}} / K_{\mathrm{I}}\right)$ for the plane strain crack approaches a constant value at large $l / a$. The values of $G_{\mathrm{ps}}$ and $G_{\mathrm{ss}}$ for the orthotropic case are somewhat larger than for the isotropic case, upon comparing the results of Fig. 6 with those of Fig. 3.

The tunnelling stress $\tau_{\mathrm{ss}}$ and the plane strain delamination stress $\tau_{\mathrm{ps}}$ are plotted against the normalised delamination length $l / a$ in Fig. 7a for selected values of $G_{\mathrm{dc}} / G_{\mathrm{mc}}$, and for $\beta=30^{\circ}$. Again, the tunnelling stress is a minimum at the point of intersection with the $\tau_{\mathrm{ps}}$ curve, and we take this minimum to define the tunnelling stress for a given value of $G_{\mathrm{dc}} / G_{\mathrm{mc}}$. The corresponding delamination length is denoted $(l / a)_{\mathrm{c}}$ as for the isotropic case. The delamination length $(l / a)_{\mathrm{c}}$ decreases and the minimum tunnelling stress increases with increasing

Table 1

Material properties of Graphite/Epoxy (T300/5208)

\begin{tabular}{lll}
\hline Material properties & Values & Unit \\
\hline$E_{L}=E_{y}$ & 181 & $\mathrm{GPa}$ \\
$E_{T}=E_{x}=E_{z}$ & 10.3 & $\mathrm{GPa}$ \\
$G_{L T}=G_{x y}$ & 7.17 & $\mathrm{GPa}$ \\
$v_{L T}=v_{y x}=v_{y z}$ & 0.28 & \\
$v_{T T}=v_{z z}$ & 0.5 & \\
\hline
\end{tabular}


$-\frac{\tau_{\mathrm{ps}}-\tau_{\mathrm{b}}}{\sqrt{\bar{E} G_{\mathrm{dc}} / a}} \quad \cdots \cdot \frac{\tau_{\mathrm{ss}}-\tau_{\mathrm{b}}}{\sqrt{E} G_{\mathrm{dc}} / a}$

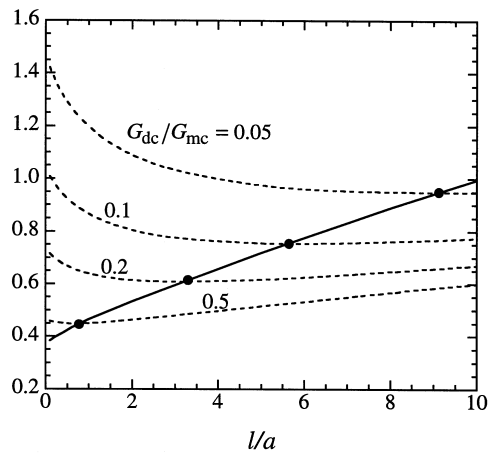

(a)

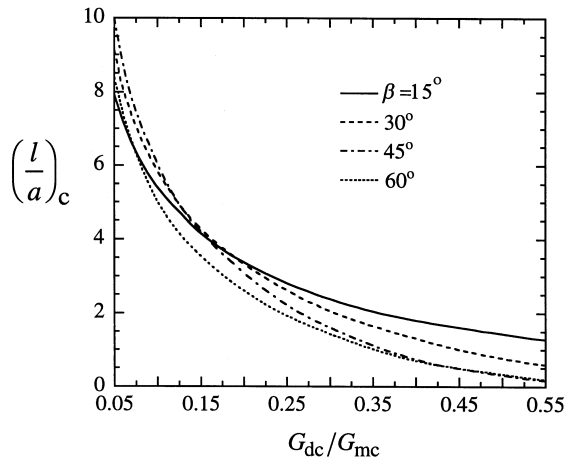

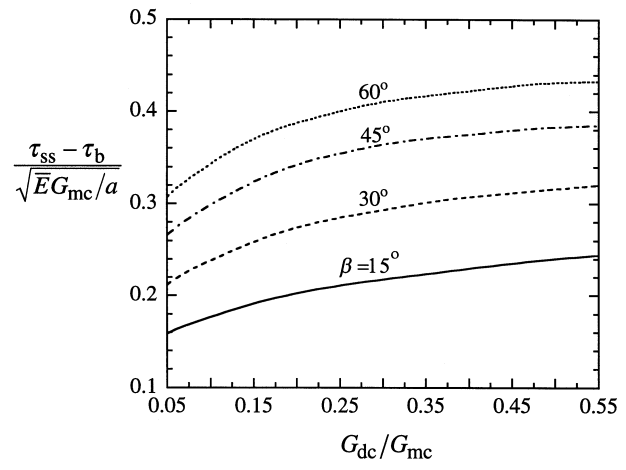

(c)

(b)

Fig. 7. (a) The applied stress $\tau_{\mathrm{ps}}$ and the tunnelling stress $\tau_{\mathrm{ss}}$ for a delamination crack in a graphiteepoxy unidirectional composite, with $\beta=30^{\circ}$; (b) Delamination length at the minimum tunnelling stress, and (c) Minimum tunnelling stress, as a function of the ratio of delamination toughness $G_{\mathrm{dc}}$ to microbuckling toughness $G_{\mathrm{mc}}$.

delamination toughness $G_{\mathrm{dc}} / G_{\mathrm{mc}}$, see Fig. $7 \mathrm{~b}$ and c. The minimum value of $\tau_{\mathrm{ss}}$ approximately doubles when $\beta$ is increased from $15^{\circ}$ to $60^{\circ}$.

The sensitivities of $(l / a)_{c}$ and of the tunnelling stress to the degree of material orthotropy are explored in Fig. 8. Here, results are given for the range of practical values $\lambda=1$ to $\lambda=20$, with $\rho=0,1$ and 5 . Recall that the isotropic case is $\lambda=$ $\rho=1$. The following broad trends can be deduced from Fig. $8 \mathrm{a}-\mathrm{d}$ taken together. The delamination length $(l / a)_{\mathrm{c}}$ becomes more sensitive to the value of $G_{\mathrm{dc}} / G_{\mathrm{mc}}$ with increasing $\lambda$ and $\rho$, and the tunnelling stress drops with increasing $\lambda$ and $\rho$. A large change in orthotropy from the isotropic case to $\lambda=20$ and $\rho=5$ has 
however only a minor effect on the delamination length $(l / a)_{\mathrm{c}}$ but a more major effect upon the associated tunnelling stress $\left(\tau_{\mathrm{ss}}-\tau_{\mathrm{b}}\right) / \sqrt{\bar{E} G_{\mathrm{mc}} / a}$.

\section{Tunnelling in a cross-ply laminate}

Practical laminated composites are usually multi-directional, with microbuckling occurring in the load-bearing $0^{\circ}$ plies. In this section, we study microbuckletunneling in a cross-ply $(90 / 0 / 90)$ laminate. The $0^{\circ}$ ply of width $2 h$ is bonded between two $90^{\circ}$ plies, as sketched in Fig. 9a. We shall use the designation [ $]_{0}$ to denote quantities such as $b_{i j}$ and $s_{i j}$ for the $0^{\circ} \mathrm{ply}$, and the designation [ $]_{90}$ for the

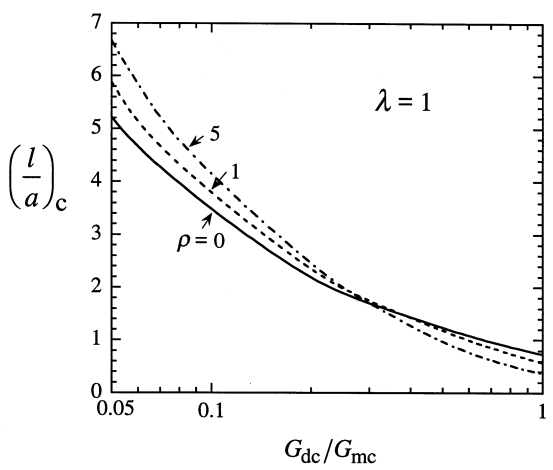

(a)

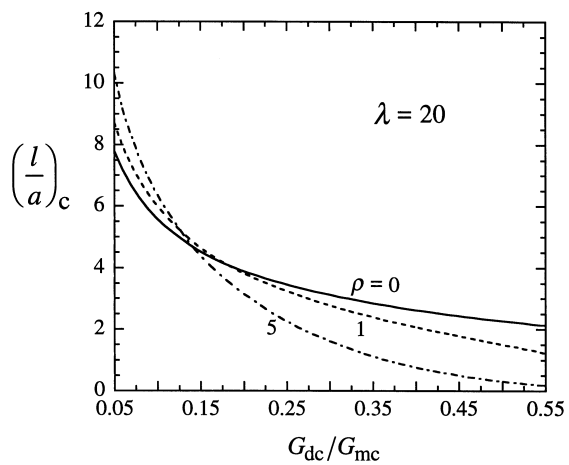

(b)

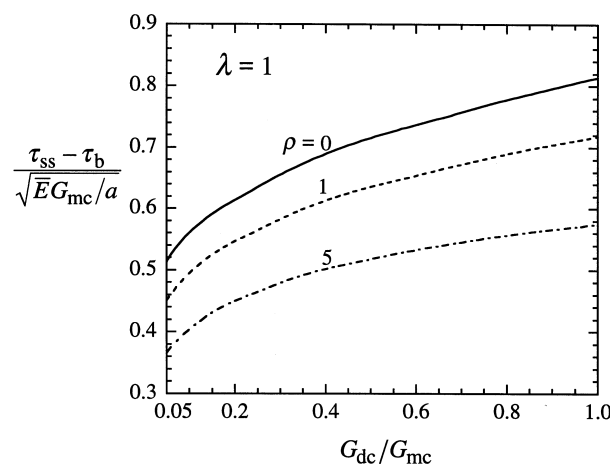

(c)

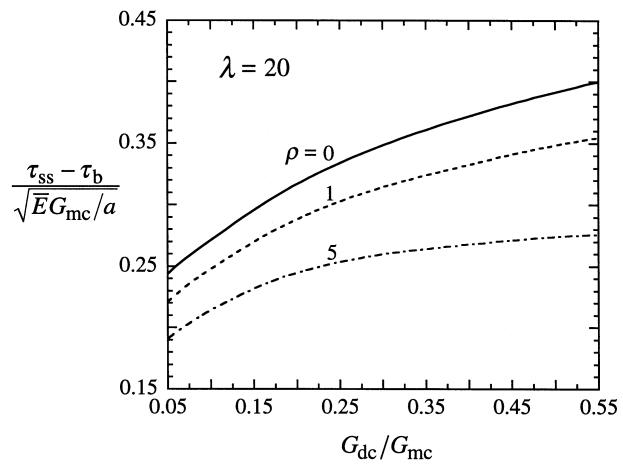

(d)

Fig. 8. Effect of orthotropy upon the delamination length and the tunnelling stress for an orthotropic laminate, with principal axes aligned with the loading directions. Delamination length at the minimum tunnelling stress for (a) $\lambda=1$ and (b) $\lambda=20$. The corresponding minimum tunnelling stress is shown for (c) $\lambda=1$ and (d) $\lambda=20$. 
$90^{\circ}$ ply. The overall width and height of the laminate are taken to be 200 times the magnitude of $h$, such that the results are representative of an infinite plate. The motivation for this study is the tunnelling of microbuckles within thick laminates. We begin by summarising the nature of the stress singularity for delamination of the cross-ply laminate.

\subsection{Crack tip field}

A delamination between the $0^{\circ}$ and $90^{\circ}$-plies is treated as an interfacial crack between dissimilar orthotropic solids. We begin by reviewing the associated crack tip field. The displacement jumps $\delta_{x}$ and $\delta_{y}$ across the delamination have the form

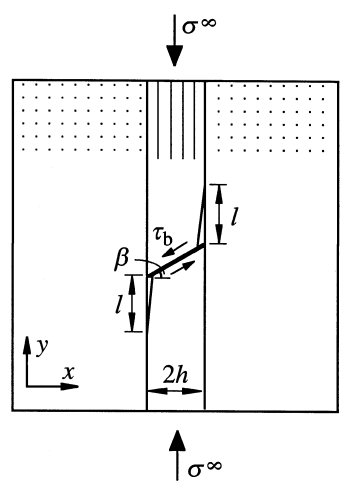

(a)

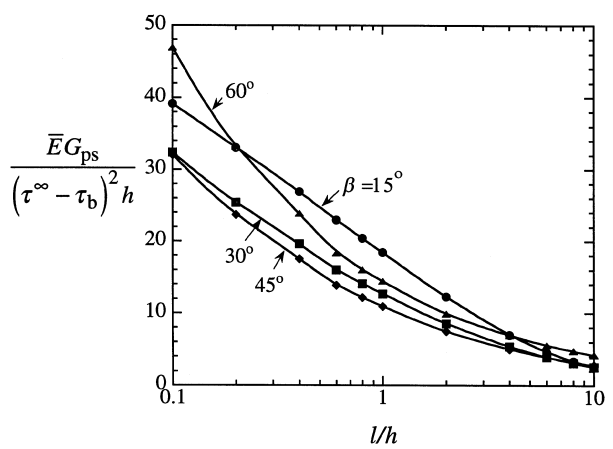

(b)

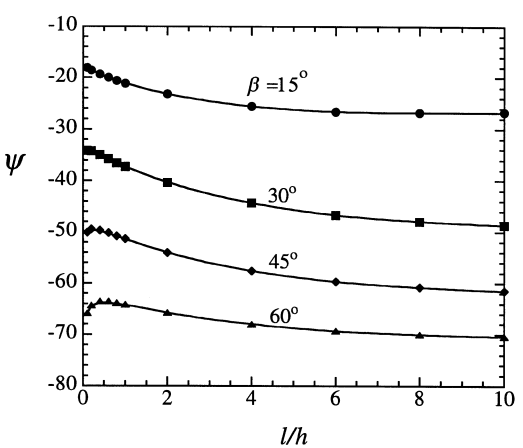

(c)

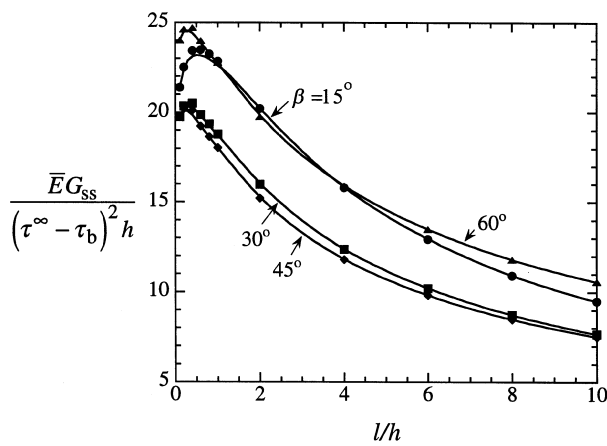

(d)

Fig. 9. Plane strain crack growth versus the tunnelling of a microbuckle in a cross-ply orthotropic composite. (a) Geometry; (b) Energy release rate $G_{\mathrm{ps}}$ for the plane strain delamination crack $\left(\bar{E} \equiv 1 /\left[b_{22}\right]_{0}\right)$; (c) Phase angle $\psi$ for the plane strain delamination crack; (d) Tunnelling energy release rate $G_{\text {ss }}$. 
(Suo, 1990b)

$$
\sqrt{\frac{H_{22}}{H_{11}}} \delta_{x}+i \delta_{y}=\frac{2 H_{11}\left(K_{\mathrm{I}}+i K_{\mathrm{II}}\right) r^{i \varepsilon}}{(1+2 i \varepsilon) \cosh (\pi \varepsilon)} \sqrt{\frac{r}{2 \pi}}
$$

where

$$
\begin{aligned}
& H_{11}=\left[2 n \lambda^{1 / 4} \sqrt{b_{11} b_{22}}\right]_{0}+\left[2 n \lambda^{1 / 4} \sqrt{b_{11} b_{22}}\right]_{90} \\
& H_{22}=\left[2 n \lambda^{-1 / 4} \sqrt{b_{11} b_{22}}\right]_{0}+\left[2 n \lambda^{-1 / 4} \sqrt{b_{11} b_{22}}\right]_{90}
\end{aligned}
$$

and $\hat{\beta}$ and $n$ are given by

$$
\sqrt{H_{11} H_{22}} \hat{\beta}=\left[\sqrt{b_{11} b_{22}}+b_{12}\right]_{0}-\left[\sqrt{b_{11} b_{22}}+b_{12}\right]_{90}
$$

and

$$
n=\sqrt{(1+\rho) / 2}
$$

Here, $\hat{\beta}$ is a generalisation of one of Dundurs' parameters.

The oscillatory index $\varepsilon$ in (17) is:

$$
\varepsilon=\frac{1}{2 \pi} \ln \left(\frac{1-\hat{\beta}}{1+\hat{\beta}}\right)
$$

The Irwin-type energy release rate is:

$$
G_{\mathrm{ps}}=\frac{H_{11}\left(K_{\mathrm{I}}^{2}+K_{\mathrm{II}}^{2}\right)}{4 \cosh ^{2}(\pi \varepsilon)}
$$

For convenience, the phase angle $\psi$ of the interface crack is defined in relation to the half width $h$ of the $0^{\circ}$ ply (Rice, 1988; Hutchinson and Suo, 1992) by

$$
\psi=\arctan \left[\frac{\operatorname{Re}\left(\mathbf{K} h^{i \varepsilon}\right)}{\operatorname{Im}\left(\mathbf{K} h^{i \varepsilon}\right)}\right]
$$

where $\mathbf{K}=K_{\mathrm{I}}+i K_{\mathrm{II}}$.

\subsection{Tunnelling analysis}

For both plane strain cracking and tunnelling the driving stress is the applied shear traction $\tau^{\infty}$ on the inclined mode II crack within the $0^{\circ}$ ply. We consider the case where the sandwiched $0^{\circ}$ ply is sufficiently thin in comparison with the neighbouring $90^{\circ}$ plies that the stress $\sigma_{22}$ in the $90^{\circ}$ plies equals the remote compressive stress $\sigma^{\infty}$. With plane strain conditions prevailing, the remote stress 
in the $0^{\circ}$ ply is uniaxial compression of magnitude

$$
\left[\sigma_{22}\right]_{0}=\frac{\left[b_{22}\right]_{90}}{\left[b_{22}\right]_{0}} \sigma^{\infty}
$$

and the resolved shear traction on the cracking plane $\tau^{\infty}$ is given by

$$
\tau^{\infty}=\left[\sigma_{22}\right]_{0} \cos \beta \sin \beta=\frac{\left[b_{22}\right]_{90} \cos \beta \sin \beta}{\left[b_{22}\right]_{0}} \sigma^{\infty}
$$

In presenting results for plane strain crack growth and for microbuckle tunnelling, the relations Eqs. (3)-(11) remain valid, with $\tau^{\infty}$ given by (23) and $\bar{E}$ given by $1 /\left[b_{22}\right]_{0}$.

\subsection{Finite element results and discussion}

The finite element mesh for the cross-ply laminate consisted of between 1900 and 3600 plane strain isoparametric elements depending on the ratio $l / h$. The elements are 8-noded quadrilateral except for some 6-noded triangular elements on the mode II crack surfaces. As for the previous cases, the square root singularity of the crack tip stress field is modelled by moving the mid-side nodes on the side adjacent to the crack tip to the quarter-points for all crack tip elements. Fixed and roller boundary conditions are imposed at the two lower corner points of the mesh in order to prevent rigid body motion. Nodal loading is applied to the faces of the mode II crack by the imposition of uniform normal and shear tractions by resolving the axial stress $\left[\sigma_{22}\right]_{0}$ on to the cracking plane.

The energy release rate $G_{\mathrm{ps}}$ of the interfacial delamination cracks is evaluated by the Parks (1974) virtual crack extension technique. The mode-mix $\psi$ was deduced from the displacement field in the vicinity of the crack tip. The average sliding displacement $\bar{\delta}$ of the mode II crack is calculated from the distribution of slip along the faces of the mode II crack. Once $\bar{\delta}$ has been found, the non-dimensional function $f$ is evaluated from Eq. (3) and the tunnelling stress is given by Eq. (11).

In the remainder of this section, we present results for a cross-ply $(90 / 0 / 90)$ laminate made from a Graphite-Epoxy composite, with properties specified in Table 1 . The computed values of energy release rate $G_{\mathrm{ps}}$ and mode-mix $\psi$ are plotted in Fig. $9 \mathrm{~b}$ and c, respectively, as a function of the delamination length $l / h$, for selected values of the inclination $\beta$. As for the homogeneous case of the unidirectional Graphite/Epoxy, $G_{\mathrm{ps}}$ decreases monotonically with increasing $l / h$, and the mode mix $\psi$ is relatively insensitive to the value of $l / h$. The energy release rate $G_{\mathrm{ps}}$ is only weakly dependent upon the inclination $\beta$, whereas the mode mix is strongly dependent upon $\beta$. The tunnelling energy release rate for the cross-ply material is summarised in Fig. 9d by plotting a non-dimensional measure of $G_{\mathrm{ss}}$ against $l / h$, for $\beta$ in the range $15-60^{\circ}$. As noted previously for the homogeneous isotropic and orthotropic cases, $G_{\mathrm{ss}}$ attains a maximum at $l / h \approx 1$.

The remote stresses for plane strain cracking $\tau_{\mathrm{ps}}$ and for tunnelling $\tau_{\mathrm{ss}}$ are plotted against $l / h$ in Fig. 10, for selected values of the normalised delamination 
energy $G_{\mathrm{dc}} / G_{\mathrm{mc}}$, with $\beta=30^{\circ}$. As for the homogeneous cases, $\tau_{\mathrm{ss}}$ attains a minimum at the intersection point $\tau_{\mathrm{ss}}=\tau_{\mathrm{ps}}$. The minimum value of $\tau_{\mathrm{ss}}$ and the associated non-dimensional delamination length $(l / h)_{\mathrm{c}}$ are shown in Fig. 10b and c: as expected $(l / h)_{\mathrm{c}}$ drops and $\tau_{\mathrm{ss}}$ increases with increasing $G_{\mathrm{dc}} / G_{\mathrm{mc}}$. The effect of inclination $\beta$ upon $(l / h)_{\mathrm{c}}$ and upon $\tau_{\mathrm{ss}}$ is minor.

\section{Concluding discussion}

In this study we have determined the plane strain cracking stress $\tau_{\mathrm{ps}}$ and the

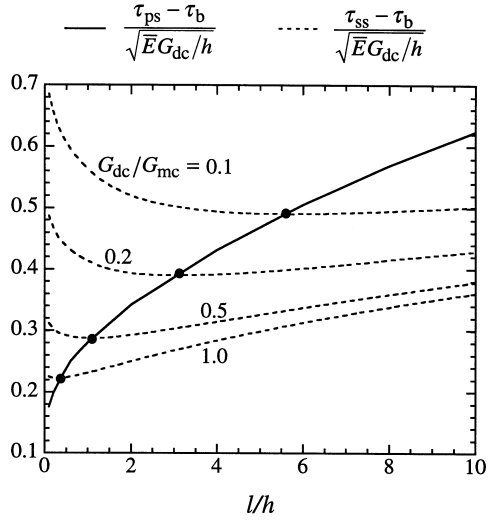

(a)

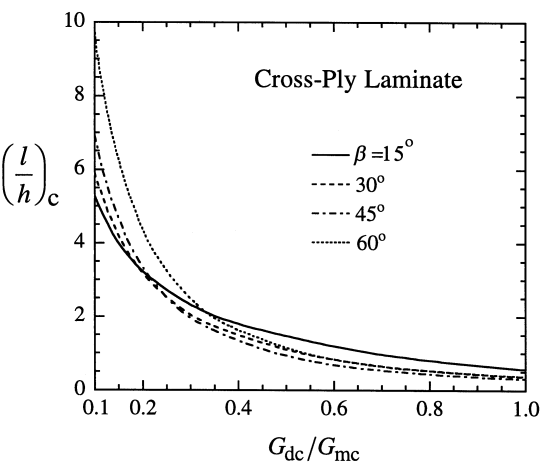

(b)

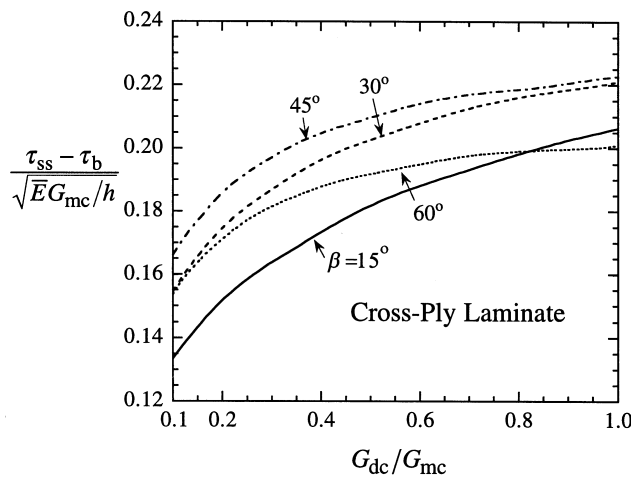

(c)

Fig. 10. (a) The applied stress $\tau_{\mathrm{ps}}$ and the tunnelling stress $\tau_{\mathrm{ss}}$ for a delamination crack in a cross-ply orthotropic composite with $\beta=30^{\circ}$; (b) Delamination length at the minimum tunnelling stress, and (c) Minimum tunnelling stress, as a function of the ratio of delamination toughness $G_{\mathrm{dc}}$ to microbuckling toughness $G_{\mathrm{mc}}$. 
tunnelling stress $\tau_{\mathrm{ss}}$ for a wide range of unidirectional fibre composites, and for a specific example of a cross-ply composite. To date, systematic experimental studies of tunnelling by fibre microbuckling have not been reported in the literature, and it is hoped that the present theoretical study will stimulate future experimental investigations into this phenomenon. The sensitivities of the delamination length $(l / a)_{\mathrm{c}}$ and of tunnelling $\tau_{\mathrm{ss}}$ to the type of laminate and to the ratio of delamination toughness to microbuckling toughness are summarised in Fig. 11, for the case $\beta=30^{\circ}$. The delamination length $(l / a)_{\mathrm{c}}$ has only a weak dependence upon the type of composite whereas the tunnelling stress $\tau_{\mathrm{ss}}$ is sensitive to the fibre architecture: the magnitude of $\tau_{\mathrm{ss}}$ for an isotropic solid is 2 to 4 times that for orthotropic solids and for a cross-ply laminate.

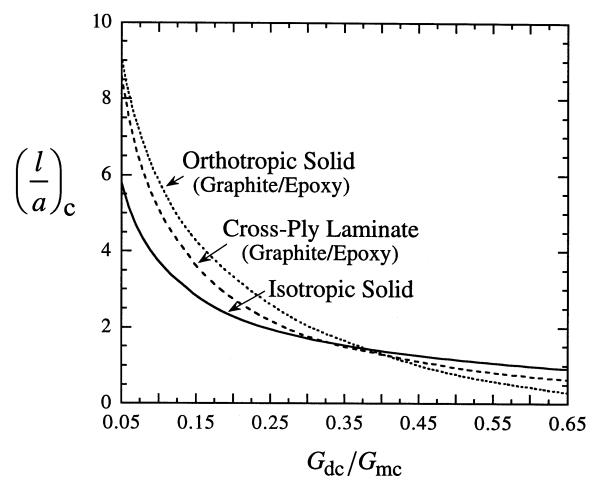

(a)

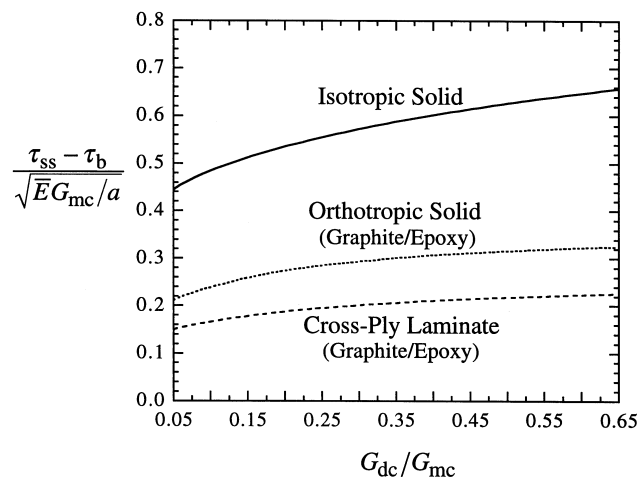

(b)

Fig. 11. Comparison of tunnelling solutions for various lay-ups of composite, with $\beta=30^{\circ}$. (a) Delamination length at the minimum tunnelling stress, and (b) Minimum tunnelling stress, as a function of the ratio of delamination toughness $G_{\mathrm{dc}}$ to microbuckling toughness $G_{\mathrm{mc}}$. 
It is instructive to use the above analysis to estimate the magnitude of the tunnelling stress $\tau_{\mathrm{ss}}$ in a cross-ply carbon-epoxy laminate. Consider again the cross-ply (90/0/90) laminate made from the Graphite-Epoxy composite, with properties specified in Table 1. The delamination toughness is estimated to be about $1 \mathrm{~kJ} \mathrm{~m}^{-2}$ (Gibson, 1994) and the crack bridging strength associated with microbuckle broadening is assumed to be of the order of the shear strength of the composite at large shear strains, $\tau_{\mathrm{b}} \approx 50 \mathrm{MPa}$ (Budiansky et al., 1998). The mode II toughness associated with microbuckling is estimated from (44) of Budiansky et al. (1998) to be

$$
G_{\mathrm{mc}} \approx \frac{5}{8} \pi d \beta^{3 / 2} \cos \beta \sqrt{\tau_{Y} \bar{E}}
$$

where $d$ is the fibre diameter, $\tau_{Y}$ is the longitudinal shear strength of the composite, and $\bar{E}$ is the longitudinal plane strain modulus of the unidirectional composite undergoing microbuckling. On taking an inclination $\beta=30^{\circ}, \tau_{Y}=50$ $\mathrm{MPa}, \bar{E}=182 \mathrm{GPa}$ and $d=5.5 \mu \mathrm{m}$, the relation (24) gives $G_{\mathrm{mc}}=10.7 \mathrm{kJm}^{-2}$. Thus, $G_{\mathrm{dc}} / G_{\mathrm{mc}}$ equals 0.1 , and from Fig. $11 \mathrm{a}, \mathrm{b}$ the delamination crack length $(l / a)_{\mathrm{c}}$ equals 5 and $\left(\tau_{\mathrm{ss}}-\tau_{\mathrm{b}}\right) / \sqrt{\bar{E} G_{\mathrm{mc}} / a}$ equals 0.16 . On taking a mid-ply of thickness such that $a=0.5 \mathrm{~mm}$, we obtain $\tau_{\mathrm{ss}}=366 \mathrm{MPa}$, and thus $\left[\sigma_{\mathrm{ss}}\right]_{0}=\left[\sigma_{22}\right]_{0}=$ $\tau_{\mathrm{ss}} /(\cos \beta \sin \beta)=845 \mathrm{MPa}$ in the $0^{\circ} \mathrm{ply}$. This value is about one half of the typical initiation strength of a unidirectional laminate.

We conclude by addressing the issue of how to prevent the steady state tunnelling of a microbuckle. It is clear from Fig. $11 \mathrm{~b}$ that the tunnelling stress $\sigma_{\mathrm{ss}}$ is elevated by ensuring a large delamination toughness $G_{\mathrm{dc}}$, a large microbuckling toughness $G_{\mathrm{mc}}$ and thin layers within the composite. Through-thickness stitching is a promising technique for raising $G_{\mathrm{dc}}$, the use of thick fibres elevates $G_{\mathrm{mc}}$ (see relation (24)), and the use of thin plies reduces the magnitude of $a$.

\section{Acknowledgements}

The authors are grateful to Dr. Y.D.S. Rajapakse (US Office of Naval Research, grant 0014-91-J-1916) and to the EPSRC for financial support.

\section{Appendix A. Integral equation formulation and solution procedure}

In order to check the accuracy of the finite element calculations, an integral formulation was also used in the isotropic case.

\section{A.1. Formulation of integral equations}

The solution for an isolated edge dislocation in an infinite solid is used to solve the isotropic plane strain elasticity problem of Fig. 2b. A system of integral equations is formed by representing both the mode II crack and the anti- 
symmetric delamination cracks by distributions of dislocations. With reference to Fig. 2b, let $A(\eta)$ denote the amplitude of the dislocation shearing distribution from $-a$ to $a$ along the mode II crack line, and let $A_{2 x}(\xi)$ and $A_{2 y}(\xi)$ denote the amplitude of the dislocation opening and shearing distributions from 0 to $l$ along the delamination-crack line.

By the principle of superposition, the resultant shear stress along the mode II crack due to presence of the dislocations and due to the remote stress $\tau^{\infty}$ equals the constant bridging stress $-\tau_{\mathrm{b}}$, and can be written as (Chan et al., 1993; Lu, 1996)

$$
\begin{aligned}
& -\tau^{\infty}+\int_{-a}^{a} A\left(\eta^{\prime}\right) H_{1}\left(\eta, \eta^{\prime}\right) \mathrm{d} \eta^{\prime}+\int_{0}^{l} A_{2 x}(\xi) H_{2}(\eta, \xi) \mathrm{d} \xi \\
& \quad+\int_{0}^{l} A_{2 y}(\xi) H_{3}(\eta, \xi) \mathrm{d} \xi \\
& =-\tau_{\mathrm{b}}
\end{aligned}
$$

Here, $H_{1}\left(\eta, \eta^{\prime}\right)$ denotes the shear stress at a location $\eta$ along the mode II crack line due to a shearing edge dislocation at $\eta^{\prime}$; similarly, $H_{2}(\eta, \xi)$ and $H_{3}(\eta, \xi)$ denote the shear stresses at $\eta$ due to an opening edge dislocation at $\xi$ in the $x$ direction, and to a shearing edge dislocation at $\xi$ in the $y$-direction. The Green's functions $H_{2}(\eta, \xi)$ and $H_{3}(\eta, \xi)$ include the contributions from edge dislocations along the lower delamination crack as well as the upper delamination crack, with the appropriate symmetry conditions imposed.

Similarly, the traction-free condition along the delamination crack line for $0<$ $\xi<l$ is:

$$
\begin{aligned}
& \int_{-a}^{a} A(\eta) H_{4}(\xi, \eta) \mathrm{d} \eta+\int_{0}^{l} A_{2 x}\left(\xi^{\prime}\right) H_{5}\left(\xi, \xi^{\prime}\right) \mathrm{d} \xi^{\prime}+\int_{0}^{l} A_{2 y}\left(\xi^{\prime}\right) H_{6}\left(\xi, \xi^{\prime}\right) \mathrm{d} \xi^{\prime} \\
& \quad=0 \\
& \int_{-a}^{a} A(\eta) H_{7}(\xi, \eta) \mathrm{d} \eta+\int_{0}^{l} A_{2 x}\left(\xi^{\prime}\right) H_{8}\left(\xi, \xi^{\prime}\right) \mathrm{d} \xi^{\prime} \\
& \quad+\int_{0}^{l} A_{2 y}\left(\xi^{\prime}\right) H_{9}\left(\xi, \xi^{\prime}\right) \mathrm{d} \xi^{\prime} \\
& \quad=0
\end{aligned}
$$

where $H_{4}(\xi, \eta)$ and $H_{7}(\xi, \eta)$ denote respectively the normal stress $\sigma_{x x}(\xi)$ and the shear stress $\sigma_{x y}(\xi)$ along the delamination crack line due to a unit edge shearing dislocation at a location $\eta$ on the inclined crack plane. The Green's functions $H_{5}\left(\xi, \xi^{\prime}\right)$ and $H_{6}\left(\xi, \xi^{\prime}\right)$ denote the contributions to $\sigma_{x x}(\xi)$ from an opening edge dislocation at $\xi^{\prime}$ in the $x$-direction, and from a shearing edge dislocation in the $y$ direction, respectively. $H_{8}\left(\xi, \xi^{\prime}\right)$ and $H_{9}\left(\xi, \xi^{\prime}\right)$ denote the contributions to $\sigma_{x y}(\xi)$ 
from an opening edge dislocation at $\xi^{\prime}$ in the $x$-direction, and from a shearing edge dislocation in the $y$-direction, respectively. In constructing $H_{5}, H_{6}, H_{8}$ and $H_{9}$, anti-symmetric distributions of dislocation density are assumed on the upper and lower delamination cracks.

The net displacement jump for the whole crack vanishes, implying that

$$
\int_{-a}^{a} A(\eta) \mathrm{d} \eta=0
$$

and

$$
\sin \beta \int_{0}^{l} A_{2 x}(\xi) \mathrm{d} \xi-\cos \beta \int_{0}^{l} A_{2 y}(\xi) \mathrm{d} \xi=0
$$

Note that Eq. (A3b) is a statement that the accumulated displacement jump at the end of each delamination crack is constrained to give only sliding of the inclined mode II crack, without opening.

\section{A.2. Solution procedure}

The kernels of the above integral equations have Cauchy singularities, and require appropriate evaluation. The dislocation distributions are expressed by Chebyshev polynomials of the first kind,

$$
\begin{aligned}
& A(\eta)=A(t)=\frac{1}{\sqrt{1-t^{2}}} \sum_{k=0}^{N} C_{k} T_{k}(t) \\
& A_{2 x}(\xi)=A_{2 x}(s)=\frac{1}{\sqrt{1-s^{2}}} \sum_{k=0}^{N} D_{k} T_{k}(s) \\
& A_{2 y}(\xi)=A_{2 y}(s)=\frac{1}{\sqrt{1-s^{2}}} \sum_{k=0}^{N} E_{k} T_{k}(s)
\end{aligned}
$$

where the following changes of variable have been introduced,

$$
\eta=a t \text { for }|t|<1, \quad \text { and } \quad \xi=\frac{(1+s)}{2} l \text { for }|s|<1 .
$$

With the choice (A4), the Erdogan and Gupta (1972) integration scheme can be employed to solve the $3 N+3$ unknown real coefficients $\left(C_{k}, D_{k}, E_{k}\right)$ in Eq. (A4). We note that the dislocation densities in Eq. (A4) generate square-root stress singularities at each end of the crack line segments (at $|t|=1$ and $|s|=1$ ). At the delamination crack tips such square-root singularities exist. In contrast, at the kinks between the mode II crack and the delamination cracks, algebraic stress 
singularities exist but are of lower order than the usual square root crack-type. The functional forms stated in Eq. (A4) do not capture the correct singularities at the kinks. However, numerical experimentation revealed that the nature of the assumed asymptotic stress state at the kinks has a negligible effect on the calculated energy release rates for plane strain cracking and for tunnelling. The calculations reported here were performed by allowing crack tip singularities to exist at the kinks, but with the two closure conditions (A3) imposed. The $3 N+3$ unknown coefficients in Eq. (A4) are determined by enforcing (A1) at $N+1$ Gauss-Chebyshev collocation points on the mode II crack surface and by enforcing Eq. (A2) at $N$ Gauss-Chebyshev collocation points on the delamination crack surface. Additional calculations were performed by enforcing the singularities at the kinks to vanish: this led to a change in the calculated energy release rate for tunnelling and plane strain crack advance by less than $1 \%$ over the full parameter range considered.

Once a solution has been obtained, the stress intensity factors and the energy release rate of the delamination crack tip are calculated by

$$
\begin{aligned}
& K_{\mathrm{I}}=-\frac{E}{8 \pi\left(1-v^{2}\right)} \pi \sqrt{2 \pi l} \sum_{k=0}^{N} D_{k} \\
& K_{\mathrm{II}}=\frac{E}{8 \pi\left(1-v^{2}\right)} \pi \sqrt{2 \pi l} \sum_{k=0}^{N} E_{k}
\end{aligned}
$$

and

$$
G_{\mathrm{ps}}=\frac{\left(1-v^{2}\right)}{E}\left(K_{\mathrm{I}}^{2}+K_{\mathrm{II}}^{2}\right)
$$

where $E$ is Young's modulus and $v$ is the Poisson's ratio.

The relative sliding displacement at any point $\eta$ of the mode II crack is given by

$$
\delta(\eta)=\cos \beta \int_{0}^{l} A_{2 x}(\xi) \mathrm{d} \xi+\sin \beta \int_{0}^{l} A_{2 y}(\xi) \mathrm{d} \xi+\int_{\eta}^{a} A\left(\eta^{\prime}\right) \mathrm{d} \eta^{\prime}
$$

\section{References}

ABAQUS manual, version 5.7. Hibbitt, Karlsson and Sorensen, Inc., 1997.

Beuth, J.R., 1992. Cracking of thin bonded films in residual tension. Int. J. Solids Struct. 29, 16571675 .

Budiansky, B., Fleck, N.A., 1993. Compressive failure of fibre composites. J. Mechanics and Physics of Solids 41 (1), 183-211.

Budiansky, B., Fleck, N.A., Amazigo, J.C., 1998. On kink-band propagation in fibre composites. J. Mech. Phys. Solids 46 (9), 1637-1653. 
Chan, K.S., He, M.Y., Hutchinson, J.W., 1993. Cracking and stress redistribution in ceramic layered composites. Mat. Sci. Eng. A167, 57-64.

Chaplin, C.R., 1977. Comressive fracture in unidirectional glass-reinforced plastics. J. Mat. Sci. 12, $347-352$.

Cox, B.N., Marshall, D.B., 1996. Crack initiation in fiber-reinforced brittle laminates. J. Amer. Ceram. Soc. 79 (5), 1181-1188.

Erdogan, F., Gupta, G.D., 1972. On the numerical solution of singular integral equations. Q. J. Applied Maths 30, 525-534.

Evans, A.G., Adler, W.F., 1978. Kinking as a mode of structural degradation in carbon fiber composites. Acta Metall. 26, 725-738.

Fleck, N.A., 1997. Compressive failure of fibre composites. Advances in Applied Mechanics 33, 43-113.

Fleck, N.A., Deng, L., Budiansky, B., 1995. Prediction of kink width in compressed fibre composites. J. Applied Mechanics 62, 329-337.

Fleck, N.A., Sivashankar, S., Sutcliffe, M.P.F., 1997. Compressive failure of composites due to microbuckle growth (special issue). Eur. J. Mech. A/Solids, 1665-82.

Gibson, R.F., 1994. Principles of Composite Material Mechanics. McGraw-Hill, New York.

Ho, S., Suo, Z., 1993. Tunnelling cracks in constrained layers. ASME J. Appl. Mech. 60, 890-894.

Hsu, S.Y., Vogler, T.J., Kyriakides, S., 1998. Compressive strength predictions for fibre composites. J. Applied Mechanics 65, 7-16.

Hutchinson, J.W., Suo, Z., 1992. Mixed mode cracking in layered materials. Advances in Applied Mechanics 29, 63-191.

Jensen, H.M., 1999. Analysis of compressive failure of layered materials by kink band broadening. Int. J. Solids and Structures 36 (23), 3427-3441.

Jensen, H.M., Christoffersen, J., 1997. Kink band formation in fibre reinforced materials. J. Mech. Phys. Solids 45 (7), 1121-1136.

Kyriakides, S., Arseculeratne, R., Perry, E.J., Liechti, K.M., 1995. On the compressive failure of fibre reinforced composites. Int. J. Solids and Structures 32 (6/7), 689-738.

Kyriakides, S., Ruff, A.E., 1997. Aspects of failure and postfailure of fibre composites in compression. J. Comp. Materials 31, 2000-2037.

Lauterbach, B., Gross, D., 1998. Crack growth in brittle solids under compression. Mechanics of Materials 29 (8), 81-92.

Lekhnitskii, S.G., 1963. Theory of Elasticity of an Anisotropic Body. Holden-Day, San Francisco.

Lu, T.J., 1996. Crack branching in all-oxide ceramic composites. J. Am. Ceram. Soc. 79, 266-274.

Matos, P.P.L., McMeeking, R.M., Charalambides, P.G., Dory, M.D., 1989. A method for calculating stress intensities in bimaterial fracture. Int. J. Fract. 40, 235-254.

Moran, P.M., Liu, X.H., Shih, C.F., 1995. Kink band formation and band broadening in fibre composites under compressive loading. Acta Metall. et Mater. 43, 2943-2958.

Nemat-Nasser, S., Horii, H., 1982. Compression-induced nonplanar crack extension with application to splitting, exfoliation, and rockburst. J. Geo. Res. 87, 6805-6821.

Palmer, A.C., Rice, J.R., 1973. The growth of slip surfaces in the progressive failure of overconsolidated clay. Proc. Roy. Soc. Lond. A332, 527-548.

Parks, D.M., 1974. A stiffness derivative finite element technique for determination of crack tip stress intensity factors. Int. J. Fract. 10, 487-501.

Rice, J.R., 1988. Elastic fracture mechanics concepts for interfacial cracks. ASME J. Appl. Mech. 55, 98-103.

Rosen, B.W. 1965. Mechanics of composite strengthening, chapter 3 in Fiber composite materials. American Society of Metals, chapter 3, 37-75.

Schapery, R.A., 1995. Prediction of compressive strength and kink bands in composites using a work potential. Int. J. Solids Structures 32 (6/7), 739-765.

Schultheisz, C.R., Waas, A.M., 1996. Compressive failure of composites, Part I: testing and micromechanical theories. Prog. Aerospace Sci. 32, 1-42.

Sivashankar, S., Fleck, N.A., Sutcliffe, M.P.E., 1996. Microbuckle propagation in a unidirectional carbon fibre-epoxy matrix composite. Acta Mater. 44 (7), 2581-2590. 
Soutis, C., Fleck, N.A., Smith, P.A., 1991. Failure prediction technique for compression loaded carbon fibre-epoxy laminate with open holes. J. Composite Material 25, 1476-1498.

Soutis, C., Curtis, P.T., Fleck, N.A., 1993. Compressive failure of notched carbon fibre composites. Proc. R. Soc. Lond. A440, 241-256.

Steif, P.S., 1984. Crack extension under compressive loading. Engng. Fract. Mech. 30, 463-473.

Suo, Z., 1990a. Delamination specimens for orthotropic materials. ASME J. Appl. Mech. 57, 627-634.

Suo, Z., 1990b. Singularities, interfaces and cracks in dissimilar anisotropic media. Proc. R. Soc. Lond. A427, 331-358.

Tsai, S.W., 1988. Composites Design, 4th. ed. Think Composites Publ, Dayton, Ohio, USA.

Vogler, T.J., Kyriakides, S., 1997. Initiation and axial propagation of kink bands in fibre composites. Acta Mater. 45 (6), 2443-2454.

Waas, A.M., Schultheisz, C.R., 1996. Compressive failure of composites, Part II: experimental studies. Prog. Aerospace Sci. 32, 1-42. 